\title{
Detecção de metalo-beta-lactamases em amostras hospitalares de Pseudomonas aeruginosa e Acinetobacter baumannii
}

\author{
Detection of metallo-beta-lactamases in hospital strains of Pseudomonas aeruginosa and Acinetobacter \\ baumannii
}

Deuseli Quaresma de Figueiredo; ; Luciana Fundão S. Castro²; Kátia Regina N. Santos³; Lúcia Martins Teixeira4; Silvia Susana B. de Mondino ${ }^{5}$

\section{unitermos \\ Pseudomonas aeruginosa \\ Acinetobacter baumannii \\ Detecção de \\ metalo-beta-lactamase}

\section{resumo}

Introdução e objetivo: Entre os bacilos Gram-negativos não fermentadores da glicose, Pseudomonas aeruginosa e Acinetobacter baumannii são os agentes etiológicos de infecções hospitalares mais frequentes. A resistência aos carbapenemas entre esses patógenos está se tornando um problema terapêutico mundial e a produção de metalo-beta-lactamases ( $\mathrm{MBL}$ ) tem emergido como um dos mecanismos responsáveis por esta resistência. O presente estudo objetivou verificar a produção de MBL em amostras de P. aeruginosa e A. baumannii isoladas de pacientes internados no Hospital Estadual Azevedo Lima, localizado em Niterói-RJ. Material e métodos: Um total de 400 amostras (286 P. aeruginosa e 114 A. baumannii) foi identificado por meio de sistemas comerciais e sua susceptibilidade aos antimicrobianos foi testada por disco-difusão. As amostras resistentes a ceftazidima foram submetidas à avaliação da produção de $\mathrm{MBL}$ pelo método de disco-aproximação. Resultados: A produção de $\mathrm{MBL}$ foi verificada em 49 amostras de $P$. aeruginosa (17,1\%) e em 20 amostras de $A$. baumannii $(17,5 \%)$. Grande parte das amostras de $P$. aeruginosa e de $A$. baumannii foi resistente a todos os antimicrobianos testados. Conclusão: A produção de $\mathrm{MBL}$ é um dos mecanismos de resistência aos carbapenemas e outros beta-lactâmicos entre amostras de $P$. aeruginosa e $A$. baumannii isoladas no hospital-alvo desta investigação. A rápida detecção deste fenótipo de resistência é essencial para implementar medidas rígidas de controle de infecção e permitir o início de terapia empírica adequada. abstract

Introduction and objective: P. aeruginosa and A. baumannii are the most prevalent etiological agents of hospital infections among non-fermentative gram-negative bacilli. Carbapenem resistance among these pathogens has become a therapeutic problem worldwide. Metallo-beta-lactamases (MBL) production has emerged as one of the mechanisms responsible for this resistance. The aim of the present study was to assess MBL production in clinical isolates of P. aeruginosa and A. baumannii from inpatients at Hospital Estadual Azevedo Lima, Niterói, RJ. Material and methods: A total of 400 strains of P. aeruginosa (286) and A. baumannii (114) were identified through commercial systems. Antimicrobial susceptibility was tested by disk diffusion. Isolates resistant to ceftazidime were screened for $M B L$ production by disk approximation test. Results: MBL production was detected in 49 (17.1\%) P. aeruginosa isolates and in 20 (17.5\%) A. baumannii isolates. Most $\mathrm{P}$. aeruginosa and $\mathrm{A}$. baumannii samples were resistant to all antimicrobial agents. Conclusion: Production of $M B L$ is one of the mechanisms of carbapenem resistance and other beta-lactams among P. aeruginosa and A. baumannii isolates in the investigated hospital. The rapid detection of this resistance phenotype is essential to implement strict infection control procedures and initiate appropriate empirical therapy.

1. Mestre em Patologia Clínica (análises clínicas); farmacêutica-bioquímica do Hospital Estadual Azevedo Lima (HEAL) e da Vigilância Sanitária de Niterói.

2. Acadêmica do curso de Biomedicina da Universidade Federal Fluminense (UFF); bolsista do Programa Institucional de Bolsas de Iniciação Científica do Conselho Nacional de Desenvolvimento Científico e Tecnológico (PIBIC/CNPq).

3. Doutor em Ciências (microbiologia); professor associado do Departamento de Microbiologia Médica do Instituto de Microbiologia Professor Paulo de Cóes da Universidade Federal do Rio de Janeiro (UFR)).

4. Doutor em Ciências (microbiologia); professor titular do Departamento de Microbiologia Médica do Instituto de Microbiologia Professor Paulo de Cóes da UFR).

5. Doutor em Ciências (microbiologia); professor adjunto do Departamento de Patologia da Faculdade de Medicina da UFF.

Trabalho realizado nos laboratórios de Microbiologia do Serviço de Análises Clínicas do HEAL e do Hospital Universitário Antônio Pedro da Universidade Federal Fluminense (HUAP/UFF); e nos Laboratórios de Apoio Biotecnológico e de Infecções Hospitalares do Instituto de Microbiologia Professor Paulo de Cóes da UFR). 


\section{Introdução}

Pseudomonas aeruginosa e Acinetobacter baumannii são bactérias Gram-negativas, não-fermentadoras da glicose, amplamente distribuídas na natureza e no ambiente hospitalar, que apresentam necessidades nutricionais mínimas e tolerância a condições físicas variadas (umidade, temperatura, $\mathrm{pH}$ ), o que favorece sua sobrevivência em superfícies úmidas e secas por períodos prolongados. Apresentam também grande capacidade de desenvolver resistência aos antimicrobianos, assim como escapar dos mecanismos de defesa do hospedeiro. Podem estar presentes em alimentos (frutas e vegetais frescos) e colonizar a pele de seres humanos saudáveis e outros sítios úmidos do corpo, incluindo orofaringe, mucosa nasal, axilas e períneo ${ }^{(13,15)}$.

As infecções hospitalares por $P$. aeruginosa e $A$. baumannii geralmente envolvem os tratos respiratório e urinário, além de feridas, incluindo sítios de cateterização, podendo progredir para septicemia. Os fatores de risco para infecções incluem permanência prolongada em unidades de tratamento intensivo (UTIs), procedimentos invasivos, imunossupressão decorrente do uso de quimioterápicos para o tratamento de neoplasias, tratamento com antimicrobianos, ventilação mecânica, queimaduras, feridas cirúrgicas e existência de doenças de base ${ }^{(2,36)}$.

Os carbapenemas têm sido uma das drogas de escolha para o tratamento de infecções hospitalares causadas por bacilos Gram-negativos devido ao amplo espectro de ação bactericida e à sua grande estabilidade frente à maioria das beta-lactamases, inclusive as de espectro ampliado - Extended-Spectrum Beta-Lactamases (ESBL) ${ }^{(42)}$. Entretanto, a resistência aos carbapenemas tem sido observada principalmente em $P$. aeruginosa e $A$. baumannii, em decorrência de mecanismos, como diminuição da permeabilidade da membrana externa por perda ou alterações na estrutura de porinas; atividade de bombas de efluxo que promovem a diminuição da concentração do antimicrobiano no interior da bactéria; e/ou por ação de beta-lactamases (carbapenemases). Diversas beta-lactamases adquiridas pertencentes à classe $B$ de Ambler e conhecidas como metalo-beta-lactamases (MBL), ou à classe $D$, também conhecidas como oxacilinases, já foram identificadas nestes patógenos ${ }^{(11,23,29,31)}$.

Atualmente, são conhecidas seis subclasses de MBL adquiridas: imipenemase (IMP), descoberta no Japão(27); Verona imipenemase (VIM), descoberta na Itália, em 1997, em uma amostra clínica de $P$. aeruginos ${ }^{(18)}$; São Paulo metalo-beta-lactamase (SPM-1), isolada em 1997 de amostra clínica de $P$. aeruginosa no Hospital São Paulo da Universidade Federal de São Paulo
(HSP/UNIFESP) ${ }^{(40)}$; German imipenemase (GIM-1), descoberta em amostras clínicas de $P$. aeruginosa isoladas na Alemanha ${ }^{(5)}$; Seoul imipenemase (SIM-1), detectada em sete amostras clínicas de A. baumannii provenientes da Coreia, entre 2003 e 2004 (21); e Australian imipenemase (AIM), descoberta em uma amostra de $P$. aeruginosa isolada em 2007, na Austrália ${ }^{(12)}$.

No Brasil, o primeiro relato da ocorrência de amostras de P. aeruginosa produtoras de MBL ocorreu em 2002, no Hospital Universitário Clementino Fraga Filho, da Universidade Federal do Rio de Janeiro (HUCFF/UFRJ) ${ }^{(28)}$. No mesmo ano também houve registro da ocorrência de $P$. aeruginosa produtora de $M B L$ na região Nordeste, em João Pessoa $(P B)^{(34)}$. Os tipos de MBL detectados no Brasil são SPM, IMP e VIM. As do tipo SPM são as mais frequentemente encontradas e têm-se mantido restritas ao Brasil, sendo detectadas em São Paulo (SP), Salvador (BA), Curitiba, Londrina e Maringá (PR), Brasília (DF), Santo André (SP) e Fortaleza (CE) ${ }^{(10)}$. As IMP foram detectadas nas cidades de Brasília(25), São Paulo(3), Rio de Janeiro(4) e Porto Alegre ${ }^{(44)}$ e as VIM, em São Paulo ${ }^{(33)}$. Entre as amostras de $A$. baumannii, foram detectadas apenas as do tipo IMP, na cidade de São Paulo( ${ }^{(9,39)}$.

As MBLs caracterizam-se por necessitarem de dois íons divalentes, usualmente zinco $\left(\mathrm{Zn}^{++}\right)$, como cofator para a reação de hidrólise do anel beta-lactâmico, podendo ser detectadas por meio de testes fenotípicos com o auxílio de um agente quelante, como os ácidos etilenodiaminotetracético (EDTA) e 2-mercaptopropiônico (MPA), entre outros ${ }^{(3,42)}$. Nos testes de disco difusão, utilizados para avaliar a susceptibilidade aos antimicrobianos, geralmente as amostras produtoras de MBL são resistentes à ceftazidima, enquanto a susceptibilidade aos carbapenemas pode ser variada ${ }^{(26)}$.

\section{Objetivo}

O presente estudo teve por objetivo verificar a produção de MBL em amostras de $P$. aeruginosa e A. baumannii isoladas de pacientes internados no Hospital Estadual Azevedo Lima (HEAL), em Niterói, entre os anos de 2005 e 2006, bem como documentar a suscetibilidade aos antimicrobianos entre estas amostras.

\section{Material e métodos}

\section{Amostras bacterianas}

No período de janeiro de 2005 a dezembro de 2006, foram avaliadas 400 amostras, sendo 286 de $P$. aeruginosa 
e 114 de $A$. baumannii, isoladas de diferentes espécimes clínicos, provenientes de pacientes internados nos diferentes setores do HEAL, que possui atendimento terciário, com cerca de 200 leitos ativos. Apenas uma amostra por paciente foi incluída neste estudo.

\section{Identificação das amostras}

As amostras foram identificadas utilizando-se painéis ID $32 \mathrm{~N}$ do sistema MicroScan, autoSCAN-4 (Dade Behring). A partir da verificação da pureza das culturas foram realizados testes complementares à identificação, antes e depois da utilização do sistema comercial de identificação, citado anteriormente. No caso das amostras de $P$. aeruginosa, os testes executados foram observação das características morfo-tintoriais após coloração pelo método de Gram; teste da oxidase; teste de crescimento em caldo a $42^{\circ} \mathrm{C}$; e observação da produção de pigmentos. Nas amostras de A. baumannii, os testes executados foram observação das características morfo-tintoriais após coloração pelo método de Gram; teste da oxidase; teste da catalase; teste de crescimento em caldo a $44^{\circ} \mathrm{C}$; e observação da produção de hemólise em ágar sangue ${ }^{(35,17)}$.

\section{Teste de susceptibibilidade aos antimicrobianos}

Foi utilizado o método de disco-difusão em ágar (Kirby-Bauer), seguindo-se as recomendações do Clinical and Laboratory Standards Institute $(C L S I)^{(6)}$. Os antimicrobianos testados foram amicacina (AMI, $30 \mu \mathrm{g}$ ); aztreonam (ATM, $30 \mu \mathrm{g})$; cefepima (CPM, $30 \mu \mathrm{g})$; ceftazidima (CAZ, $30 \mu \mathrm{g})$; ciprofloxacina (CIP, $5 \mu \mathrm{g})$; gentamicina (GEN, $10 \mu \mathrm{g})$; imipenem (IMP, $10 \mu \mathrm{g})$; meropenem (MER, $10 \mu \mathrm{g}$ ); piperacilina-tazobactam (PPT, 100/10 $\mu \mathrm{g}$ ); e tobramicina (TOB, $30 \mu \mathrm{g})$. Nas amostras de $A$. baumannii foram testados também ampicilina-sulbactam (ASB, 10/10 $\mu \mathrm{g})$; tetraciclina (TET, $30 \mu \mathrm{g}$ ); e sulfametoxazol-trimetoprim (SUT, 23,75/1,25 $\mu \mathrm{g}$ ). Apesar de não haver padronização para o teste de susceptibilidade à polimixina $\mathrm{B}(\mathrm{POL}, 300 \mathrm{U})$ pelo método de disco-difusão, o mesmo foi realizado e a interpretação do diâmetro dos halos de inibição foi realizada conforme protocolo sugerido anteriormente ${ }^{(8)}$. Os discos de antimicrobianos foram obtidos da Cecon (São Paulo, Brasil). As amostras-controle utilizadas foram $P$. aeruginosa ATCC 27853 e Escherichia coli ATCC 25922.

\section{Detecção de produção de MBL}

As amostras de $P$. aeruginosa e $A$. baumannii resistentes a ceftazidima foram submetidas à avaliação da produção de MBL por meio do método de disco-aproximação, de acordo com os protocolos propostos por Arakawa et al.(1) e Lee et al.(19), com algumas modificações. Resumidamente, cerca de três colônias foram utilizadas para o preparo das suspensões bacterianas em salina estéril, com turvação correspondente ao padrão 0,5 da escala de McFarland. Essas suspensões foram semeadas com o auxílio de swabs estéreis, em placas contendo meio de ágar Mueller-Hinton (Difco). Em seguida, discos contendo ceftazidima $(30 \mu \mathrm{g})$, imipenem $(10 \mu \mathrm{g})$ e discos estéreis de papel de filtro adicionados de $3 \mu \mathrm{l}$ de uma solução de MPA (1,2 $\mathrm{g} / \mathrm{ml}$, Merck) e de $10 \mu \mathrm{l}$ de uma solução $0,5 \mathrm{M}$ de EDTA $(\mathrm{pH}=8)$ foram posicionados na placa. As distâncias (centro a centro) entre os discos de antimicrobianos e os discos de papel de filtro, que receberam os agentes quelantes utilizados, foram de $1,5 \mathrm{~cm}$ (disco contendo EDTA) e de $2 \mathrm{~cm}$ (disco contendo MPA) (Figura 1). As placas foram incubadas em aerobiose a $35^{\circ} \mathrm{C}$ por 16 a 18 horas. $\mathrm{A}$ observação do surgimento de um halo ou do aumento do tamanho do halo ao redor dos discos de ceftazidima e/ou imipenem, localizados próximos ao disco com MPA e/ou com EDTA, indicou teste positivo para detecção da produção de MBL. A não-observação deste evento indicou teste negativo. Foram incluídas cepas-padrão de $P$. aeruginosa produtoras das enzimas SPM-1 (P1088) e IMP-1 (98-P4978) e de A. baumannii produtora da enzima IMP-1 (48-695) (Figura 2).

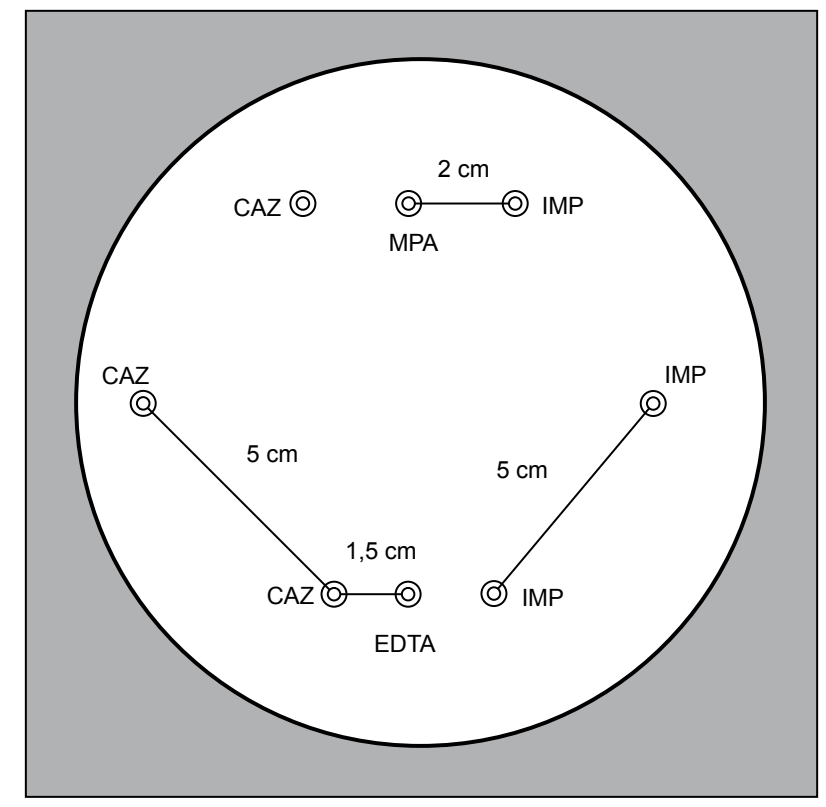

Figura1 - Representação esquemática da disposição, em placa contendo ágar Mueller Hinton, dos discos contendo os agentes quelantes e antimicrobianos utilizados no teste fenotípico de detecção de MBL pelo método de disco aproximação 


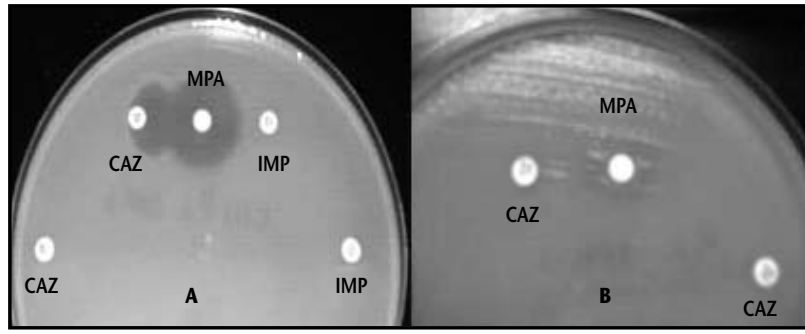

Figura 2 - Ilustração de resultados de teste fenotípico para detecção de MBL com amostra de $P$. aeruginosa (método de disco aproximação)

A: teste positivo; B: teste negativo.

\section{Resultados}

Todas as amostras de $P$. aeruginosa e A. baumannii apresentaram taxas variadas de resistência à maioria dos antimicrobianos testada. Como podem ser observadas na Tabela 1, as taxas de resistência à maioria dos antimicrobianos testada foram significativamente maiores entre as amostras de $A$. baumannii quando comparadas com aquelas observadas entre as amostras de $P$. aeruginosa. Todas as amostras foram sensíveis à polimixina $B$.

A detecção de MBL foi verificada em 49 amostras de $P$. aeruginosa $(17,1 \%)$ e 20 amostras de $A$. baumannii $(17,5 \%)$. Vinte e nove amostras de $P$. aeruginosa $(59,2 \%)$ e 14 amostras de A. baumannii MBL (+) (70\%) foram detectadas indistintamente pelos testes realizados com os dois agentes quelantes utilizados (EDTA e MPA). Os resultados dos testes para detecção de MBL, de acordo com o agente quelante utilizado, podem ser observados na Tabela 2.

A maior parte das amostras de $P$. aeruginosa $\mathrm{MBL}(+)$ foi isolada de urina $(23 ; 48 \%)$, seguida de espécimes do trato respiratório inferior (TRI): aspirado traqueal $(10 ; 20 \%)$ e lavado broncoalveolar (2; 4\%); sangue $(3 ; 6 \%)$ e ponta de cateter ( $3 ; 6 \%)$; secreção de ferida operatória (2; 4\%) e secreção de úlcera de compressão $(2 ; 4 \%)$; e outras fontes de isolamento $(4 ; 8 \%)$, como secreção auricular, secreção uretral, secreção de coto de amputação e secreção de dreno abdominal. Com relação às amostras de $A$. baumannii $\operatorname{MBL}(+)$, foi verificada a mesma frequência de isolamento em urina e sangue $(5 ; 25 \%)$, aspirado traqueal $(4 ; 20 \%)$, secreção de sítio cirúrgico $(3 ; 15 \%)$, ponta de cateter (2; $10 \%)$ e swab retal $(1 ; 5 \%)$. O setor de internação onde ocorreu o maior número de isolamento de cepas de $P$. aeruginosa $\mathrm{MBL}(+)$ foi a Unidade de Pacientes Graves (28; $57 \%)$, seguida do Centro de Tratamento Intensivo (CTI) (7; $14,2 \%)$, das enfermarias de Curta Permanência $(5 ; 10,2 \%)$, Clínica Médica $(4 ; 8,4 \%)$, Longa Permanência $(3 ; 6 \%)$ e Traumatologia $(2 ; 4,2 \%)$. Os setores de onde foi isolado o maior número de amostras de $A$. baumannii $\mathrm{MBL}(+)$ foram a Unidade de Pacientes Graves (7; 35\%) e a enfermaria de Clínica Médica (7; 35\%), seguidas da enfermaria de Longa Permanência ( $3 ; 15 \%)$, da Unidade de Terapia Intensiva
Tabela 1

\begin{tabular}{lcr} 
Tabela 1 & Estadual Azevedo Lima, no período de \\
\hline Agentes antimicrobianos & P. aeruginosa $\mathrm{n}^{0}(\%)$ & A. ba \\
Amicacina & $77(26,9)$ \\
Aztreonam & $115(40,2)$ \\
Cefepima & $121(42,3)$ \\
Ceftazidima & $116(40,6)$ \\
Ciprofloxacina & $159(55,6)$ \\
Gentamicina & $155(54,2)$ \\
Imipenem & $93(35,6)$ \\
Meropenem & $79(27,7)$ \\
Piperacilina-tazobactam & $80(28)$ \\
Tobramicina & $157(54,9)$ \\
Ampicilina-sulbactam & - \\
Tetraciclina & - \\
Sulfametoxazol-trimetoprima & - \\
(-): não-testado. & \\
Foram considerados significativos valores de $p$ iguais ou inferiores a $0,05$.
\end{tabular}

Comparação da resistência aos antimicrobianos entre $\mathbf{2 8 6}$ amostras de $\boldsymbol{P}$. aeruginosa e 114 amostras de $A$. baumannif isoladas de pacientes internados no Hospital Estadual Azevedo Lima, no período de janeiro de $\mathbf{2 0 0 5}$ a dezembro de 2006

$\begin{array}{ccc}\text { P. aeruginosa } \mathrm{n}^{0}(\%) & \text { A. baumannii } \mathrm{n}^{0}(\%) & p \\ 77(26,9) & 79(69,3) & <0,0001 \\ 115(40,2) & 100(87,7) & <0,0001 \\ 121(42,3) & 94(82,5) & <0,0001 \\ 116(40,6) & 98(86) & <0,0001 \\ 159(55,6) & 97(85,1) & <0,0001 \\ 155(54,2) & 69(60,5) & 0,6 \\ 93(35,6) & 50(43,9) & 0,032 \\ 79(27,7) & 57(50) & <0,0001 \\ 80(28) & 78(68,4) & <0,0001 \\ 157(54,9) & 66(57,9) & 0,6 \\ - & 21(18,4) & - \\ - & 32(28,1) & -\end{array}$


Resultados dos testes para detecção

de MBL em amostras de $P$. aeruginosa

e $A$. baumannit, de acordo com 0

Tabela 2 agente quelante utilizado

$\begin{array}{cccc}\begin{array}{c}\text { Cepas MBL } \\ (+)\end{array} & \text { MPA e EDTA } & \text { MPA } & \text { EDTA } \\ \text { P. aeruginosa } & 29(59,2 \%) & 14(28,6 \%) & 6(12,2 \%) \\ \text { A. baumannii } & 14(70 \%) & 3(15 \%) & 3(15 \%)\end{array}$

MBL: metalo-beta-lactamases; MPA: ácido 2-mercaptopropiônico; EDTA: ácido etilenodiaminotetracético.
Entretanto, resistência a este antimicrobiano já foi relatada tanto entre amostras de $P$. aeruginosa ${ }^{(11,31)}$ como entre amostras de $A$. baumannii( ${ }^{(39)}$. É importante ressaltar que a metodologia recomendada pelo CLSI 2009 para avaliar susceptibilidade à polimixina $B$ em amostras de $P$. aeruginosa e $A$. baumannii é o teste de diluição, com a determinação da concentração inibitória mínima (CIM). Essa metodologia não foi utilizada neste trabalho, portanto futuros estudos deverão ser realizados para confirmar os resultados obtidos pelo teste de disco difusão.

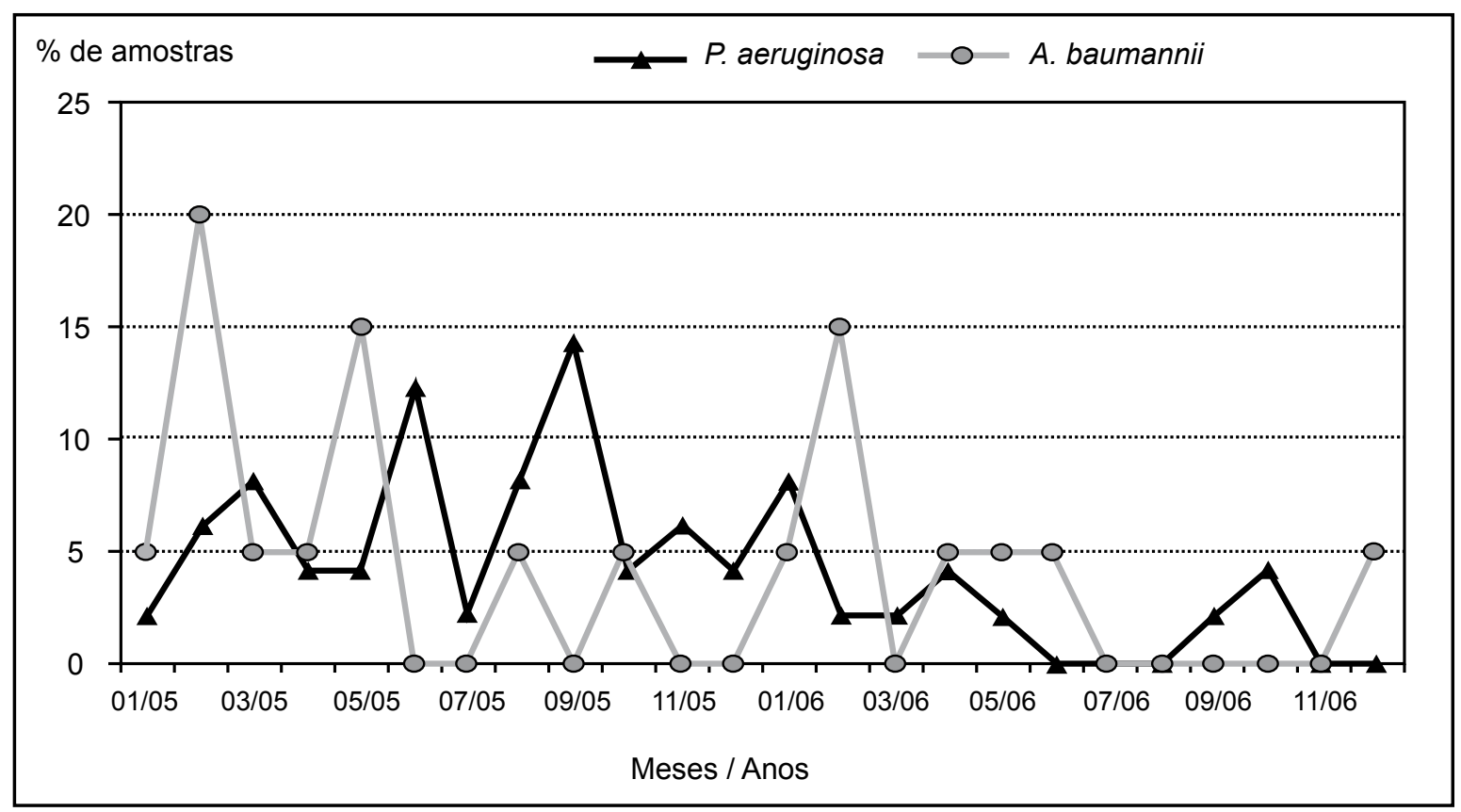

Figura 3 - Distribuição temporal do isolamento de 49 amostras de P. aeruginosa e 20 amostras de A. baumannii produtoras de MBL, isoladas de pacientes internados no Hospital Azevedo Lima no período de janeiro de 2005 a dezembro de 2006

Neonatal (UTI neonatal) $(2 ; 10 \%)$ e da enfermaria de Curta Permanência $(1 ; 5 \%)$.

A maioria $(69,6 \%)$ das amostras MBL (+) foi isolada no ano de 2005, ao passo que em 2006 o isolamento foi de $30,4 \%$ (Figura 3).

\section{Discussão}

O monitoramento da susceptibilidade aos antimicrobianos entre amostras hospitalares de $P$. aeruginosa e de A. baumannii é uma ferramenta de extrema importância, auxiliando na escolha de esquemas adequados para tratamento de infecções por estes agentes. Em concordância com os dados da literatura, todas as amostras avaliadas no presente estudo foram sensíveis à polimixina $B^{(16,24,44)}$.
Entre as amostras de A. baumannii, as taxas de sensibilidade mais elevadas foram detectadas frente à ampicilina-sulbactam $(81,6 \%)$ e à tetraciclina (71,9\%). É interessante destacar que, de acordo com alguns autores, o sulbactam apresenta efeito antimicrobiano direto contra A. baumannii ${ }^{(7,22)}$. Esse dado reforça a utilidade da ampicilina-sulbactam no tratamento de infecções causadas por A. baumannii no hospital do estudo. As tetraciclinas (doxiciclina e minociclina) podem ser úteis em alguns casos, como quando a amostra apresenta resistência à ampicilina-sulbactam e aos carbapenemas; a tigeciclina também pode ser uma boa opção, já que os mecanismos de resistência que inativam as tetraciclinas, de um modo geral, não agem sobre esta droga ${ }^{(29)}$. Quanto às amostras de $P$. aeruginosa, os antimicrobianos para os quais apresentaram as taxas de sensibilidade mais elevadas foram amicacina $(73,1 \%)$, piperacilina-tazobactam (72\%), meropenem (72,3\%) e imipe- 
nem $(67,4 \%)$, o que os torna boas escolhas para o tratamento de infecções por este microrganismo, no hospital avaliado. Karlowsky et al.(15), em estudo realizado nos Estados Unidos, encontraram taxas de sensibilidade mais elevadas para esses antimicrobianos, variando de $80 \%$ a $90 \%$.

A taxa de resistência aos carbapenemas verificada neste estudo entre as amostras de $P$. aeruginosa foi de $30,2 \%$. No Brasil, as taxas de resistência aos carbapenemas observadas em estudos anteriores com $P$. aeruginosa são, em geral, elevadas, variando entre $15 \%$ e $65 \%(4,16,24,28,33,44)$. As taxas de resistência aos carbapenemas detectadas entre as amostras de $A$. baumannii avaliadas ( $43,9 \%$ ao imipenem e $50 \%$ ao meropenem) foram bastante elevadas, quando comparadas com as verificadas por Gales et al. ${ }^{(8)}$ (8\% ao imipenem e $10,7 \%$ ao meropenem) e Tognim et al. ${ }^{(38)}$ em estudo realizado em hospitais da América Latina, que foram de 13,1\% e 13,2\%, respectivamente.

A produção de MBL em amostras de $P$. aeruginosa está se tornando frequente no Brasil e em outros países da América Latina $^{(10,4)}$. No presente estudo, $17,1 \%$ das amostras de $P$. aeruginosa foram produtoras de MBL. Resultados semeIhantes foram obtidos por Pellegrino et al. ${ }^{(28)}$ entre amostras isoladas na cidade de Rio de Janeiro $(15,5 \%)$ e por Gales et al. ${ }^{(10)}$ em estudo realizado em São Paulo $(19,7 \%)$. Em outros estudos realizados em hospitais no Brasil, foram detectados percentuais menores: $2 \%\left({ }^{(34)}, 5,5 \%{ }^{(37)}\right.$ e $7,7 \%{ }^{(41)}$. Lee et al. ${ }^{(20)}$, em estudo realizado na Coreia com amostras de $P$. aeruginosa isoladas em 1997, detectaram taxas inferiores $(11,1 \%)$, próximas àquelas reportadas pelo estudo SENTRY, em hospitais do Japão (10,8\%). Este mesmo estudo detectou taxas mais elevadas na Itália $(39,1 \%)^{(14)}$. Com relação às amostras de $A$. baumannii, $17,5 \%$ foram produtoras de MBL. No Brasil, tem sido raro o encontro de cepas de $A$. baumannii produtoras de $M B L^{(32)}$. Nessa espécie, parece predominar a ocorrência de carbapenemases do grupo $D$ adquiridas ${ }^{(30)}$.

De acordo com nossos resultados, a utilização concomitante de ambos os agentes quelantes no teste de discoaproximação parece ser a metodologia mais adequada para detectar a produção de MBL, tanto em amostras de $P$. aeruginosa como de $A$. baumannii, pois algumas amostras foram detectadas somente pelos testes empregando MPA, e outras, somente por aqueles em que o EDTA foi utilizado, em concordância com outros estudos(1, 19, 24,43).

A realização de testes fenotípicos para detecção de MBL em amostras de $P$. aeruginosa e $A$. baumannii é de grande relevância para a escolha adequada do esquema terapêutico antimicrobiano, pois essas enzimas são capazes de hidrolisar a maioria dos beta-lactâmicos e nem sempre caracterizam um fenótipo de resistência aos carbapenemas ${ }^{(26)}$. Além disso, a realização desses testes para detecção de MBL em cepas de $P$. aeruginosa e A. baumannii não-sensíveis aos carbapenemas seria importante para se ter conhecimento da prevalência de amostras produtoras de MBL, o que auxiliaria a Comissão de Controle de Infecção Hospitalar (CCIH) na implementação de medidas adequadas de controle das infecções e prevenção de disseminação deste mecanismo de resistência.

A maioria das amostras $\mathrm{MBL}(+)$ foi isolada do trato urinário e do TRI, principalmente de pacientes internados na Unidade de Pacientes Graves. O tempo de internação prolongado, a realização frequente de procedimentos invasivos e o uso intenso de antimicrobianos de amplo espectro, neste setor, constituem fatores predisponentes para a aquisição de infecções por amostras multirresistentes ${ }^{(2,36)}$.

A redução na taxa de isolamento de amostras $\mathrm{MBL}(+)$, ocorrida em 2006, com relação ao ano de 2005, pode ser explicada, pelo menos em parte, pela implementação, pela $\mathrm{CClH}$ do HEAL, de medidas rígidas de controle de infecções, após a divulgação dos resultados parciais do estudo, ao final de 2005.

Estudos adicionais serão realizados com as amostras $\mathrm{MBL}(+)$, para a sua caracterização, incluindo a identificação dos determinantes genéticos envolvidos.

\section{Conclusão}

O estudo mostrou que $17,1 \%$ e $17,5 \%$, respectivamente, das amostras de $P$. aeruginosa e $A$. baumannii, isoladas no HEAL no período de janeiro de 2005 a dezembro de 2006, produziram MBL. A ocorrência desse mecanismo de resistência aos beta-lactâmicos nos alerta para a necessidade de se estabelecer critérios para a utilização de antimicrobianos, principalmente os carbapenemas, no hospital estudado.

A inclusão de testes fenotípicos para a detecção da produção de MBL na rotina diagnóstica hospitalar se mostrou relevante. $\mathrm{O}$ teste de disco-aproximação, utilizado neste estudo para esse fim, empregando dois agentes quelantes, demonstrou ser de fácil execução.

\section{Agradecimentos}

Agradecemos à Dra. Ana C. Gales (Laboratório Alerta, Disciplina de Infectologia, UFSP), que gentilmente nos cedeu as cepas utilizadas como controles positivos no teste fenotípico de detecção de MBL. 


\section{Referências}

1. ARAKAWA, Y. et al. Convenient test for screening metallo- $\beta$ lactamase-producing Gram-negative bacteria by using thiol compounds. J Clin Microbiol, v. 38, n. 1, p. 40-3, 2000.

2. BLONDEL-HILL, E. et al. Pseudomonas. In: MURRAY, P. R. et al. (eds.). Man Clin Microbiol. 9. ed. Washington, DC: ASM Press, 2007. Cap. 48; p. 734-48.

3. BUSH, K. Metallo-B-lactamases: a class apart. Clin Infect Dis, v. 27, suppl. 1, p. 48-53, 1998.

4. CARVALHO, A. P. D. et al. Characterization of an epidemic carbapenem-resistant Pseudomonas aeruginosa producing SPM-1 metallo-B-lactamase in a hospital located in Rio de Janeiro, Brazil. Microb Drug Resist, v. 12, n. 2, p. 103-8, 2006.

5. CASTANHEIRA, M. et al. Molecular characterization of a betalactamase gene, blaGIM-1, encoding a new subclass of metallo-ß-lactamase. Antimicrob Agents Chemother, v. 48, n.12, p. 4654-61, 2004.

6. CLINICAL AND LABORATORY STANDARDS INSTITUTE (CLSI). Performance standards for antimicrobial susceptibility testing. Sixteenth informational supplement. CLSI document M100-S16. Wayne, PA, USA. 2006.

7. CORBELLA, X. et al. Efficacy of sulbactam alone and in combination with ampicillin in nosocomial infections caused by multiresistant Acinetobacter baumannii. J Antimicrob Chemother, v. 42, p. 793-802, 1998.

8. GALES, A. C. et al. Contemporary assessment of antimicrobial susceptibility testing methods for polymyxin B and colistin: review of available interpretative criteria and quality control guidelines. J Clin microbiol, v. 39, p. 183-90, 2001.

9. GALES, A. C. et al. Emergence of an IMP-like metallo-enzyme in an Acinetobacter baumannii clinical strain from a Brazilian teaching hospital. Diagn Microbiol Infect Dis, v. 45 , n. 1, p. 77-9, 2003 a.

10. GALES, A. C. et al. Dissemination in distinct Brazilian regions of an epidemic carbapenem-resistant Pseudomonas aeruginosa producing SPM metallo-ß-lactamase. J Antimicrob Chemother, v. 52, n. 4, p. 699-702, 2003b.

11. GALES, A. C. et al. Global assessment of the antimicrobial activity of polymyxin B against 54731 clinical isolates of Gram-negative bacilli: report from the SENTRY Antimicrobial Surveillance Program (2001-2004). Clin Microbiol Infect, v. 12, p. 315-21, 2006.

12. GUPTA, V. Metallo beta lactamases in Pseudomonas aeruginosa and Acinetobacter species. Expert Opin Investig Drugs, v. 17, n. 2, p. 131-43, 2008.

13. HOUANG, E. T. S. et al. Epidemiology and infection control implications of Acinetobacter spp. in Hong Kong. J Clin Microbiol, v. 39, p. 228-34, 2001.

14. JONES, R. N. etal. Emerging epidemic of metallo- $\beta$-lactamasemediated resistances. Diagn Microbiol Infect Dis, v. 51, p. 77-84, 2005.

15. KARLOWSKY, J. A. et al. Surveillance for antimicrobial susceptibility among clinical isolates of Pseudomonas aeruginosa and Acinetobacter baumannii from hospitalized patients in the United States, 1998 to 2001. Antimicrob Agents Chemother, v. 47, p.1681-88, 2003.
16. KOKIS, V. M. et al. Identification of an imipenem-resistant Pseudomonas aeruginosa clone among patients in a hospital in Rio de Janeiro. J Hosp Infect, v. 60, p. 19-26, 2005.

17. KONEMAN, E.W. et al. Diagnóstico microbiológico. São Paulo: Medsi, 2001.

18. LAURETTI, L. et al. Cloning and characterization of blaVIM, a new integron-borne metallo-ß-lactamase gene from a Pseudomonas aeruginosa clinical isolate. Antimicrob Agents Chemother, v. 43, n. 7, p. 1584-90, 1999.

19. LEE, K. et al. Modified Hodge and EDTA-disk synergy tests to screen metallo-B-lactamase-producing strains of Pseudomonas and Acinetobacter species. Clin Microbiol Infect, v. 7, p. 88-91, 2001.

20. LEE, K. et al. Metallo-B-lactamase-producing Gramnegative bacilli in Korean Nationwide Surveillance of Antimicrobial Resistance group hospitals in 2003: continued prevalence of VIM-producing Pseudomonas spp. and increase of IMP-producing Acinetobacter spp. Diagn Microbiol Infect Dis, v. 50, p. 51-8, 2004.

21. LEE, K. et al. Novel acquired metallo-ß-lactamase gene, blaSIM-1, in a class 1 integron from Acinetobacter baumannii clinical isolates from Korea. Antimicrob Agents Chemother, v. 49, n. 11, p. 4485-91, 2005.

22. LEVIN, A. S. Multiresistant Acinetobacter infections: a role for sulbactam combinations in overcoming an emerging worldwide problem. Clin Microbiol Infect, v. 8, n. 11, p. 144-53, 2002

23. LIVERMORE, D. M. Multiple mechanisms of antimicrobial resistance in Pseudomonas aeruginosa: Our worst nightmare? Clin Infect Dis, v. 34, n. 5, p. 634-40, 2002.

24. MARRA, A. R. et al. Bloodstream infections with metallobeta-lactamase-producing Pseudomonas aeruginosa: epidemiology, microbiology, and clinical outcomes. Antimicrob Agents Chemother, v. 50, n. 1, p. 38890, 2006.

25. MENDES R. E. et al. Integron Carrying a Novel MetalloB-Lactamase Gene, blalMP-16, and a Fused Form of Aminoglycoside-Resistant Gene aac(6)-30/aac(6)-Ib: Report from the SENTRY Antimicrobial Surveillance Program. Antimicrob Agents Chemother, v. 48, n. 12, p. 4693-702, 2004

26. OH, E. J. et al. Prevalence of metallo-ß-lactamase among Pseudomonas aeruginosa and Acinetobacter baumannil in a Korean university hospital and comparison of screening methods for detecting metallo-ß-lactamase. $J$ Microbiol Meth, v. 54, p. 411-18, 2003.

27. OSANO, E. et al. Molecular characterization of an enterobacterial metallo-ß-lactamase found in a clinical isolate of Serratia marcescens that shows imipenem resistance. Antimicrob Agents Chemother, v. 38, n. 1, p. 71-8, 1994

28. PELLEGRINO, F. L. et al. Occurrence of a multidrug-resistant Pseudomonas aeruginosa clone in different hospitals in Rio de Janeiro, Brazil. J Clin Microbiol, v. 40, n. 7, p. 2420-4, 2002. 
29. PEREZ, F. et al. Global challenge of multidrug-resistant Acinetobacter baumannii. Antimicrob Agents Chemother, v. 51, n. 10, p. 3471-84, 2007.

30. POIREL L., NORDMANN P. Carbapenem resistance in Acinetobacter baumannii: mechanisms and epidemiology. Clin Microbiol Infect, v. 12, p. 826-36, 2006.

31. REIS, A. O. et al. Polymyxin-Resistant Acinetobacter spp. isolates: what is next? Emerg Infect Dis, v. 9, n. 8, p. 1025-7, 2003.

32. SADER, H. S. et al. Dissemination and diversity of metalloB-lactamase in Latin America: report from the SENTRY Antimicrobial Surveillance Program. Int J Antimic Agents, v. 25, p. 57-61, 2005a.

33. SADER, H. S. et al. IMPs, VIMs and SPMs: the diversity of metallo-ß-lactamases produced by carbapenemresistant Pseudomonas aeruginosa in a Brazilian hospital. Clin Microb Infect, v. 11, p. 73-6, 2005b.

34. SANTOS FILHO, L. et al. Determinação da produção de metalo-B-lactamases em amostras de Pseudomonas aeruginosa isoladas em João Pessoa, Paraíba. J Bras Patol Med Lab, v. 38, p. 79-84, 2002.

35. SCHRECKENBERGER, P. C. Practical Approach to the Identification of Glucose-nonfermenting Gram-negative Bacilli. A Guide Identification. Chicago: Univ. Illnois, College of Medicine at Chicago, 2000.

36. SCHRECKENBERGER, P. C. et al. Acinetobacter, Achromobacter, Chryseobacterium, Moraxella, and Other Nonfermentative Gram-Negative Rods. In: MURRAY, P. R. et al. (eds.). Man Clin Microbiol. 9. ed. Washington, DC: ASM Press. 2007. Cap. 50; p. 770-802.

37. SOARES, M. C. S. T. Estudo de resistência aos antimicrobianos em amostras de Pseudomonas aeruginosa isoladas em hospitais da cidade de NiteróiRJ. Rio de Janeiro, 2005. Dissertação (Mestrado) - Faculdade de Medicina, Universidade Federal Fluminense.

38. TOGNIM, M. C. B. et al. Resistance trends of Acinetobacter spp in Latin America and characterization of international dissemination of multi-drug resistant strains: five-year report of the SENTRY Antimicrobial Surveillance Program. Int J Infect Dis, v. 8, p. 284-91, 2004.

39. TOGNIM, M. C. B. et al. Dissemination of IMP-1 metallobeta-lactamase-producing Acinetobacter species in a Brazilian teaching hospital. Infect Control Hosp Epidemiol, v. 27, p. 742-7, 2006.

40.TOLEMAN, M. A. et al. Molecular characterization of SPM1 , a novel metallo-ß-lactamase isolated in Latin America: report from the SENTRY Antimicrobial Surveillance Program. J Antimicrob Chemother, v. 50, n. 5, p. 6739, 2002.

41. TORRES, J. C. N. et al. Cepas de Pseudomonas spp. produtoras de metalo-betalactamase isoladas no Hospital Geral de Fortaleza. J Bras Patol Med Lab, v. 42, n. 5, p. 313-19, 2006.

42. WALSH, T. R. et al. Evaluation of a new Etest for detecting metallo-ß-lactamases in routine clinical testing. J Clin Microbiol, v. 40, n. 8, p. 2755-59, 2002.

43. YAN, J. J. et al. Comparison of the double-disk, combined disk and Etest methods for detecting metallo-ßlactamases in gram-negative bacilli. Diagn Microbiol Infect Dis, v. 49, n. 1, p. 5-11, 2004.

44. ZAVASCKI, A. P. et al. Polymyxin B for the treatment of multidrug-resistant pathogens: a critical review. J Antimicrob Chemother, v. 60, p. 1206-15, 2007. 